\title{
Qualitative Properties of Wavelets Constructed from Trigonometric B-Splines
}

\author{
Mahendra Kumar Jena
}

\begin{abstract}
Recently, in [7] wavelets have been constructed by taking trigonometric B-splines as the scaling functions. In this paper, we study some of its qualitative properties like decay, regularity and vanishing moments. In particular, we show that if the order of the scaling function is $n$ then these wavelets have n-vanishing moments, have regularity of order more than n-1 and decay to zero very fast like inverse of a polynomial.
\end{abstract}

Index Terms-Trigonometric b-spline, wavelet, decay, regularity, vanishing moments.

\section{INTRODUCTION}

Wavelets form an important field for a mathematician [1], 2], [3], [4] as well as for an engineer, especially those working in signal processing [9], [12] and computer graphics [11]. They arise as a tool for Time-Frequency localization [2]-[4]. Although, the oldest work on wavelet is by Haar [5] they are relatively new in applied mathematics and are growing at an explosive rate.

We construct wavelets by following a simple, elegant and easy to implement approach known as Multiresolution Analysis (MRA) which was introduced by Daubechies [3], Mallat [9] and Chui [2]. A MRA of $L^{2}(R)$ is a nested sequence of subspaces $\left\{V_{j}\right\}$ of $L^{2}(R)$ having some special properties. These properties lead to a direct sum decomposition of $\mathrm{L}^{2}(\mathrm{R})$ :

$$
L^{2}(R)=\oplus_{\mathrm{j}} W_{j},
$$

where $W_{j}$ is the complement of $V_{\mathrm{j}}$ in $V_{j+1}$, i.e., $V_{j+1}=V_{j} \oplus$ $W_{j}$. The single basis function whose dilations and translations span the space $W_{j}$ is called a "wavelet". Not only these wavelets form a theoretical tool but also a great tool for applications because of availability of easy to implement algorithms like Mallat algorithm [9].

In most applications wavelets are chosen according to their regularity, decay and vanishing moment properties. Regularity of a function $f$ is intimately related to the decay of its Fourier transform. In particular, if Fourier transform of a function decay like inverse of a polynomial of order $p$ then it is $\mathrm{p}$ times continuously differentiable [9]. Vanishing moment property of a wavelet is also intimately related to its approximation power. Moreover, if a wavelet has $n$ vanishing moments then it can be interpreted as a differential operator [9]. Some recent progresses in wavelets

Manuscript received April 10, 2012; revised May 20, 2012.

M. K. Jena is with the Department of Mathematics, VSS University of Technology, Odisha, Burla-768018, India (e-mail: maheny2010@yahoo.com, maheny2010@gmail.com). are generalization of Daubechies wavelets [14], wavelets from differential operators [13] and wavelets from trigonometric B-splines [7]. In this paper, we show that the wavelets constructed in [7] have sufficient decay, regularity and vanishing moment properties.

The paper consists of three sections. Section 1 is the introduction. In Section 2, construction of wavelets from trigonometric B-splines is briefly outlined. In Section 3, decay, regularity and vanishing moment properties of the wavelets are shown. A conclusion is given at the end.

\section{WAVELETS CONSTRUCTED FROM TRIGONOMETRIC B- SPLINES}

Trigonometric B-splines are first introduced by Lyche [8] using a recurrence relation. Later Jena et.al. [6] used convolution formula to define trigonometric B-splines with uniform knots. Most of the results obtained there was suitable for construction of compactly supported semiorthogonal wavelet which is carried out in a recent work [7]. The novelty in that paper is to design filters for the scale dependent wavelets.

The trigonometric B-spline $\varphi_{0}^{n}(., \alpha)$ of order $\mathrm{n}$ is defined by its Fourier transform

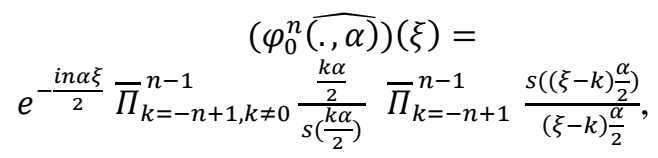

where $\bar{\Pi}_{k=-n}^{n}(y-k)=(y+n)(y+n-2) \cdots(y-n)$, $\mathrm{s}(y)=\sin (y)$ and the Fourier transform is defined by

$$
(\widehat{f(., \alpha)})(\xi)=\frac{1}{\alpha} \int_{-\infty}^{\infty} f(x, \alpha) d x
$$

The $\mathrm{j}$-th dilations of $\varphi_{0}^{n}(., \alpha)$ are defined by

$$
\varphi_{j}^{n}(x, \alpha)=\varphi_{0}^{n}\left(\frac{x}{2^{j}}, \frac{\alpha}{2^{j}}\right), j \in \mathrm{Z}
$$

and $\varphi_{j}^{n}(.-k \alpha, \alpha)$ is its k-th translation.

In the sequel, the order of B-spline is kept hidden, $s(x)$ is used for $\sin (x), c(x)$ is used for $\cos (x)$ and $z=e^{-\frac{i \xi \alpha}{2}}$.

The scaling function $\varphi_{j}^{n}(., \alpha)$ has the following two scale equation in Fourier domain

$$
\left.\left.\left(\widehat{\varphi_{J}^{n}(.}, \alpha\right)\right)(\xi)=B_{j}\left(\frac{\xi}{2}\right)\left(\varphi_{J+1}^{n(.,}, \alpha\right)\right)\left(\frac{\xi}{2}\right),
$$

where in case $n=2 m+1$ 


$$
\begin{gathered}
B_{j}\left(\frac{\xi}{2}\right)= \\
\prod_{l=1}^{n-1} \frac{1}{c\left(\frac{l \alpha}{2 j}\right)}\left(\frac{1+z}{2}\right) \Pi_{l=1}^{m}\left(\frac{1+e^{-\frac{i l \alpha}{2^{j}} z}}{2}\right) \Pi_{l=1}^{m}\left(\frac{1+e^{\frac{i l \alpha}{22^{j}} z}}{2}\right),
\end{gathered}
$$

and in case $n=2 m$

$$
\begin{gathered}
B_{j}\left(\frac{\xi}{2}\right)= \\
\prod_{l=1}^{n-1} \frac{1}{c\left(\frac{l \alpha}{2^{j}}\right)} \prod_{l=1}^{m}\left(\frac{1+e^{-\frac{i(2 l-1) \alpha}{2^{j+1}} z}}{2}\right) \Pi_{l=1}^{n-1}\left(\frac{1+e^{\frac{i(2 l-1) \alpha}{2^{j+1}} z}}{2}\right)
\end{gathered}
$$

It is shown [7] that there exist a finite filter $\left\{d_{j}(k), k \in Z\right\}$ such that the function $\psi_{j}(x, \alpha)$ defined by

$$
\psi_{j}(x, \alpha)=\frac{1}{2} \sum_{k} d_{j}(k) \varphi_{j+1}(2 x-k \alpha, \alpha)
$$

generates a wavelet basis for $W_{j}$, where $V_{j+1}=V_{j} \oplus W_{j}$. This two scale equation is expressed in Fourier domain by

$$
\left.\left.\widehat{\left(\psi_{J}^{n}(., \alpha)\right.}\right)(\xi)=D_{j}\left(\frac{\xi}{2}\right)\left(\varphi_{J+1}^{n(.,}, \alpha\right)\right)\left(\frac{\xi}{2}\right),
$$

where the Laurent polynomial $D_{j}(\xi)$ is defined by

$$
D_{j}\left(\frac{\xi}{2}\right)=\frac{1}{2} \sum_{k} d_{j}(k) e^{-\frac{i k \xi \alpha}{2}}
$$

The above filters are finite and unlike polynomial spline wavelets and some other major wavelets they do not have closed form and can't be expressed as a formula. But there construction is easy and carried out in detail in [7]. The filter $D_{j}\left(\frac{\xi}{2}\right)$ is obtained from $B_{j}\left(\frac{\xi}{2}\right)$ by the filtering technique

$$
D_{j}\left(\frac{\xi}{2}\right)=-z^{2 n} \overline{B_{\jmath}^{n}\left(\frac{\xi}{2}+\frac{\pi}{\alpha}\right)} F_{\varphi, j+1}^{n}\left(\frac{\xi}{2}+\frac{\pi}{\alpha}\right),
$$

where $\left.F_{\varphi, j+1}^{n}\left(\frac{\xi}{2}\right)=\sum_{k} \mid\left(\widehat{\varphi_{J}^{n}(., \alpha}\right)\right)\left.\left(\frac{\xi}{2}+\frac{2 \mathrm{k} \pi}{\alpha}\right)\right|^{2} \quad$ is $\quad$ the associated Euler-Frobenius polynomial. Some examples of wavelets are given in Fig. 1.

In the next section, we study some qualitative properties of wavelets constructed above.

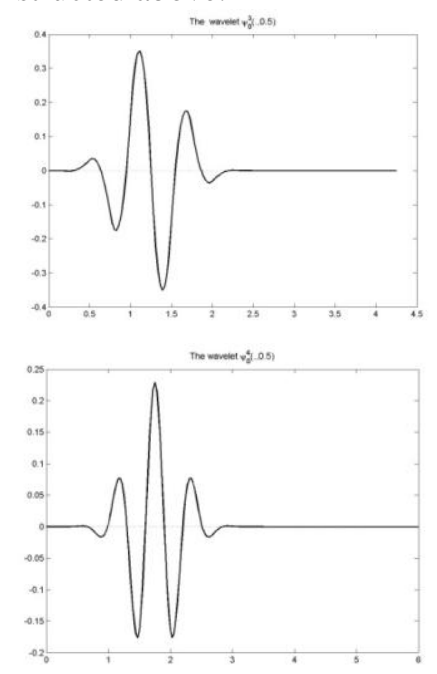

Fig. 1. The wavelet bases $\psi_{0}^{3}(., 0.5)$ and $\psi_{0}^{4}(., 0.5)$.

\section{DeCAy, Regularity, And VANishing Moments}

In this section, we show that Fourier transform of the scaling function decays like inverse of a polynomial. Further, we show that the same decay properties hold for the wavelets and these wavelets have $n$ vanishing moments. To use in the sequel, we define

$$
K(\alpha)= \begin{cases}\frac{2^{\mathrm{n}}(1 \cdot 2 \cdot 4 \cdot 6 \cdots(2 \mathrm{~m}))^{2}}{\mathrm{~s}\left(\frac{\alpha}{2}\right) \mathrm{s}^{2}\left(\frac{2 \sigma}{2}\right) \mathrm{s}^{2}\left(\frac{4 \alpha}{2}\right) \cdots \mathrm{s}^{2}\left(\frac{2 \mathrm{mg}}{2}\right)}, & n=2 m+1 \\ \frac{2^{\mathrm{n}}(1.3 .5 .7 \cdots \cdots(2 \mathrm{~m}-1))^{2}}{\mathrm{~s}^{2}\left(\frac{\alpha}{2}\right) \mathrm{s}^{2}\left(\frac{3 \alpha}{2}\right) \cdots \mathrm{s}^{2}\left(\frac{(2 \mathrm{~m}-1) \alpha}{2}\right)}, & n=2 m .\end{cases}
$$

In the above estimate $\alpha$ satisfies the stability condition [13]:

$$
\text { For no } k \text { and } j, 2^{-j} k \alpha=\pi \text {. }
$$

Theorem (10). For both $n=2 m$ and $n=2 m+1$ the scaling function $\varphi_{0}^{n}(., \alpha)$ has the following decay property:

$$
\mid\left(\varphi_{0}^{n}(\widehat{(., \alpha)})(\xi)\left|\leq K(\alpha)(1+\rho|\xi|)^{-n},\right| \xi \mid>M_{\varphi},\right.
$$

where $0<\rho \leq 1$ and $M_{\varphi}$ is any integer greater than $n$.

Proof. Observe that (see equation (1))

$$
\begin{aligned}
& \left.\left(\varphi_{0}^{n} \overline{(., \alpha}\right)\right)(\xi)= \\
& e^{-\frac{i n \xi \alpha}{2}}\left\{\begin{array}{l}
\mathrm{S}(\xi, 1) \mathrm{S}(\xi, 3) \cdots \mathrm{S}(\xi, 2 m-1), \quad n=2 m \\
\mathrm{~S}(\xi, 0) \mathrm{S}(\xi, 2) \cdots \mathrm{S}(\xi, 2 m), \quad n=2 m+1,
\end{array}\right.
\end{aligned}
$$

where for $l=1,2, \ldots, n-1$

$$
S(\xi, l)=\left(\frac{\frac{1 \alpha}{2}}{s\left(\frac{\alpha}{2}\right)}\right)^{2} \frac{s\left((\xi-l) \frac{\alpha}{2}\right)}{(\xi-l) \frac{\alpha}{2}} \frac{s\left((\xi+l) \frac{\alpha}{2}\right)}{(\xi+l) \frac{\alpha}{2}},
$$

and $S(\xi, 0)=\frac{s\left(\frac{\alpha \xi}{2}\right)}{\frac{\alpha \xi}{2}}$. The function $S(\xi, l)$ is a continuous function. Therefore, it is bounded in $\left[-M_{\varphi}, M_{\varphi}\right]$. When $|\xi|>M_{\varphi}$, we have

$$
|S(\xi, l)| \leq\left(\frac{l}{s\left(\frac{1 \alpha}{2}\right)}\right)^{2} \frac{1}{|\xi-l||\xi+l|} \leq\left(\frac{2 l}{s\left(\frac{1 \alpha}{2}\right)}\right)^{2}(1+\rho|\xi|)^{-2}
$$

where

$$
\begin{gathered}
\rho^{2}=\min \left\{1,\left(1-\frac{1}{M_{\varphi}^{2}}\right),\left(1-\frac{2^{2}}{M_{\varphi}^{2}}\right),\left(1-\frac{3^{2}}{M_{\varphi}^{2}}\right), \ldots,(1-\right. \\
\left.\frac{(n-1)^{2}}{M_{\varphi}^{2}}\right) .
\end{gathered}
$$

In case $n=2 m$ the above inequality implies

$$
\mid\left(\varphi_{0}^{n}(\widehat{., \alpha)})(\xi) \mid \leq \frac{2^{\mathrm{n}}(1.3 .5 \cdot 7 \cdots(\mathrm{n}-1))^{2}}{\left(s\left(\frac{\alpha}{2}\right) s\left(\frac{3 \alpha}{2}\right) \cdots \cdot\left(\frac{\mathrm{n}-1) \alpha}{2}\right)\right)^{2}}(1+\rho|\xi|)^{-n} .\right.
$$

This proves the theorem for the case $n=2 m$. The theorem can be proved similarly for the case $n=2 m+1$. 
Remark (11). Since there are finite numbers of filters $\mathrm{d}_{\mathrm{j}}(\mathrm{k}), \mathrm{k} \in \mathrm{Z}$ we have

$$
\mid\left(\psi_{0}^{n}(\widehat{(., \alpha)})(\xi) \mid \leq D(\alpha) K(\alpha)(1+\rho|\xi|)^{-n},\right.
$$

where $D(\alpha)=\sum_{k=0}^{3 n-2}\left|d_{j}(k)\right|$.

The above remark also shows that the bases $\varphi_{j}^{n}(., \alpha)$ and $\psi_{j}^{n}(., \alpha)$ have regularity of order more than $\mathrm{n}-1$. This is very useful in approximation theory.

In the next theorem, we show that the wavelet $\psi_{j}^{n}(., \alpha)$ has n-vanishing moments.

Theorem (12). The wavelet $\psi_{j}^{n}(., \alpha)$ for fixed $\mathrm{j}$ has $\mathrm{n}$ vanishing moments:

$$
\begin{aligned}
& n=2 m+1 . \text { For } l=1,2, \ldots, m \\
& \frac{1}{\alpha} \int_{-\infty}^{\infty} \psi_{j}^{n}(\mathrm{x}, \alpha) \mathrm{dx}=0, \\
& \frac{1}{\alpha} \int_{-\infty}^{\infty} \sin \left(2^{-(j-1)} l x\right) \psi_{j}^{n}(\mathrm{x}, \alpha) \mathrm{dx}=0, \\
& \frac{1}{\alpha} \int_{-\infty}^{\infty} \cos \left(2^{-(j-1)} l x\right) \psi_{j}^{n}(\mathrm{x}, \alpha) \mathrm{dx}=0
\end{aligned}
$$

- $\quad n=2 m$. For $l=1,2, \ldots, m$

$$
\begin{aligned}
& \frac{1}{\alpha} \int_{-\infty}^{\infty} \sin \left(2^{-j}(2 l-1) x\right) \psi_{j}^{n}(\mathrm{x}, \alpha) \mathrm{dx}=0, \\
& \frac{1}{\alpha} \int_{-\infty}^{\infty} \cos \left(2^{-j}(2 l-1) x\right) \psi_{j}^{n}(\mathrm{x}, \alpha) \mathrm{dx}=0 .
\end{aligned}
$$

Proof. We first consider the case $n=2 m+1$. Observe that the wavelet $\psi_{0}^{n}(., \alpha)$ has the two scale equation (see (7))

$$
\left.\widehat{\left(\psi_{J}^{n}\right.}(., \alpha)\right)(\xi)=D_{j}\left(\frac{\xi}{2}\right)\left(\varphi_{J+1}^{n}(., \alpha)\right)\left(\frac{\xi}{2}\right),
$$

where

$$
D_{j}\left(\frac{\xi}{2}\right)=-z^{2 n} \overline{B_{j}^{n}\left(\frac{\xi}{2}+\frac{\pi}{\alpha}\right)} F_{\varphi, j+1}^{n}\left(\frac{\xi}{2}+\frac{\pi}{\alpha}\right) .
$$

The function $F_{\varphi, j+1}^{n}(\xi)$ is always positive. Moreover, in case $n=2 m+1$ by (4)

$$
B_{j}^{n}\left(\frac{\xi}{2}\right)=0,
$$

When

Subsequently,

$$
\xi=\frac{2 \pi}{\alpha}, \frac{2 \pi}{\alpha} \pm 2^{-(j-1)} l, l=1,2, \ldots, m .
$$

$$
\left.\left(\psi_{\jmath}^{n(., \alpha}\right)\right)(\xi)=0
$$

When $\xi=0,2^{-(j-1)} l, l=1,2, \ldots, m . \quad$ Therefore, $\quad$ for $1=1,2, \ldots, m$

$$
\begin{gathered}
\frac{1}{\alpha} \int_{-\infty}^{\infty} \psi_{j}^{n}(\mathrm{x}, \alpha) \mathrm{dx}=0, \\
\frac{1}{\alpha} \int_{-\infty}^{\infty} e^{ \pm i \frac{l}{2^{j-1}} x} \psi_{j}^{n}(\mathrm{x}, \alpha) \mathrm{dx}=0
\end{gathered}
$$

From the last equation we get (17) and (18). This proves the theorem for the case $n=2 m+1$. The theorem for the case $\mathrm{n}=2 \mathrm{~m}$ is proved similarly.

The above n-vanishing moments of $\psi_{j}^{n}(., \alpha)$ is expected, since $V_{j} \perp W_{j}$ and the basis $\left\{\left(\varphi_{0}^{n}(.-l \alpha, \alpha), l \in Z\right\}\right.$ of $V_{0}$ reproduces trigonometric basis monomials of $T_{n}$, where [10]

$$
\begin{gathered}
T_{n}= \\
\left\{\begin{array}{c}
\operatorname{span}\{1, \cos (2 x), \sin (2 x), \ldots, \cos (m x), \sin (m x)\}, n= \\
2 m+1 \\
\operatorname{span}\{\cos (x), \sin (x), \ldots, \cos (m x), \sin (m x)\}, n=2 m .
\end{array}\right.
\end{gathered}
$$

We conclude that the wavelets constructed above have some good qualitative properties which make them very useful in approximation theory, reproducing trigonometric polynomials and other fields like image processing and computer graphics.

\section{REFERENCES}

[1] C. Blatter, Wavelets: A Primer, University Press (India) Private Limited, 1998.

[2] C. K. Chui, An Introduction to Wavelets, Academic Press, San Diego, California, 1992

[3] I. Daubechies, Ten Lectures on Wavelets, CBMS-NSF Series of Applied Mathematics, vol. 61, Philadelphia: SIAM, 1992.

[4] J. C. Goswami and A. K. Chan, Fundamentals of Wavelets: Theory, Algorithms, and Applications, John Wiley and Sons, Inc., New York, 1999.

[5] A. Haar, "Zur Theorie der Orthogonalen Funktionen Systeme," Mathematics Annalen vol. 69, 1910, pp. 331-371.

[6] M. K. Jena, P. Shunmugaraj, and P. C. Das, "A subdivision algorithm for trigonometric spline curves," Computer Aided Geometric Design vol. 19, 2002, pp. 71-88.

[7] M. K. Jena, "Construction of Compactly Supported Wavelets from Trigonometric B-Splines," International Journal of Wavelets, Multiresolution and Information Processing, vol. 9, no. 5, 2011, pp. 843-865.

[8] T. Lyche and R. Winther, "A stable recurrence relation for trigonometric B-splines," Journal of Approximation Theory vol. 25 , 1979, pp. 266-279.

[9] S. Mallat, A Wavelet Tour of Signal Processing, San Diego, CA: Academic, 1998.

[10] L. L. Schumaker, Spline Functions: Basic Theory, John Wiley and Sons, New York, 1980.

[11] E. J. Stollnitz, T. D. DeRose, and D. H. Salesin, Wavelets for Computer Graphics: Theory and Applications, Morgan Kaufmann, San Francisco, 1996.

[12] M. Unser, Splines: A Perfect Fit for Signal/Image Processing, to appear in IEEE Signal Processing Magazine, preprint, 1999.

[13] I. Khalidov and M. Unser, "From Differential Equations to the Construction of New Wavelet-Like Bases," IEEE Transaction on Signal Processing vol. 54, no. 4, 2006,pp. 1256-1267.

[14] C. Vonesch, T. Blu and M. Unser, "Generalized Daubechies Wavelet Families," IEEE Transaction on Signal Processing vol. 55, no. 9, 2007, pp. 4415-4429. 